\title{
Distribution of equatorial Alfvén velocity in the magnetosphere: a statistical analysis of THEMIS observations
}

\author{
Khan-Hyuk Kim ${ }^{1 *}$ (D), Gi-Jeong Kim ${ }^{1}$ and Hyuck-Jin Kwon ${ }^{2}$
}

\begin{abstract}
It has been known that the Alfvén velocity plays a significant role in generation and propagation of magnetohydrodynamic (MHD) waves. Until now, however, the global distribution of the Alfvén velocity in the magnetosphere has not been reported. To determine the spatial distribution of the Alfvén velocity, we have statistically examined the THEMIS magnetic field and electron density data obtained in the $L$ (the equatorial geocentric distance to the field line measured in Earth's radii) range of $4-12$ and at all local times near the magnetic equator between $-5^{\circ}$ and $5^{\circ}$ in magnetic latitude for 2008-2014. We observed a pronounced dawn-dusk asymmetry in the equatorial Alfvén velocity calculated from the THEMIS magnetic field and density data. That is, the dawnside Alfvén velocity is higher than the duskside Alfvén velocity. This asymmetry is due to the duskside bulge in the plasmasphere. The radial profile of the Alfvén velocity shows an increasing function of $L$ between $L=4$ and 10 in the dusk sector, while a decreasing function in the dawn sector. By comparing these Alfvén velocity distributions along the local time and radial distance, we discuss the occurrence distribution and propagation of MHD waves in the outer magnetosphere.
\end{abstract}

Keywords: Equatorial Alfvén velocity, Dawn-dusk asymmetry in the equatorial Alfvén velocity, Plasmaspheric bulge, MHD waves

\section{Introduction}

Magnetohydrodynamic (MHD) waves have become recognized as a manifestation of energy transport in the Earth's magnetosphere and serve as a useful tool for remote sensing of the magnetospheric phenomena. The MHD wave equations produce three wave modes: the shear Alfvén mode, slow mode, and fast mode. The wave packet for the shear Alfvén mode propagates only along the ambient magnetic field, while the fast mode propagates isotropically and delivers wave energy across the field lines. In the magnetosphere, the fast mode speed is similar to the Alfvén velocity except for a region of the plasma sheet, where the plasma temperature is very high (Moore et al. 1987). The Alfvén velocity is determined from magnetospheric fundamental quantities, plasma mass density, and magnetic field intensity. Since both

*Correspondence: khan@khu.ac.kr

1 School of Space Research, Kyung-Hee University, Gyeonggi, Korea

Full list of author information is available at the end of the article quantities are highly nonuniform and lead to a very inhomogeneous medium in the magnetosphere, the MHD waves change their amplitude, phase, and polarization as propagating away from the source region.

The MHD wave theory describes that the inhomogeneity in plasma density and magnetic fields enables the shear Alfvén mode and fast mode waves to be coupled to each other (Chen and Hasegawa 1974; Southwood 1974). The standing transverse Alfvén waves excited via coupling to the fast mode waves are frequently observed in near-Earth space and used to indicate field line resonance (FLR) in the magnetosphere. In the theory of FLR, the radial gradient of the Alfvén velocity is the primary factor contributing to the coupling between the fast mode waves and shear Alfvén waves (Kivelson and Southwood 1986; Zhu and Kivelson 1988). Recent theoretical and numerical studies of MHD wave propagation in the magnetosphere with a dawn-dusk asymmetric plasma density distribution have reported that strong asymmetry is introduced in the fast mode and shear Alfvén wave 
distribution (e.g., Archer et al. 2015; Degeling et al. 2018; Wright et al. 2018). Therefore, knowledge on the spatial distribution of the Alfvén velocity is important to understand how and where the MHD waves are generated and propagate.

The objective of the present study is to observationally determine the spatial distribution of the Alfvén velocity near the magnetic equator at a radial distance of $\sim 4 R_{E}$ to $12 R_{E}$ in the magnetosphere. There is a previous work for the global distribution of Alfvén velocity by Moore et al. (1987). The authors used model values of magnetic field intensity and plasma density to calculate Alfvén velocity. Unlike the previous study, we used the magnetic field and plasma density data obtained from the THEMIS spacecraft covering 7 years and statistically investigated the spatial distribution of the Alfvén velocity. We observed a strong dawn-dusk asymmetry in the Alfvén velocity. With this result, we discuss previous observations of a clear dawn-dusk asymmetry of the fast mode wave velocity (Matsuoka et al. 1995) and Pc5 wave occurrence rate (e.g., Anderson et al. 1990; Nosé et al. 1995; Takahashi et al. 2015, 2016).

\section{Data}

The primary data used in this study are (1) the total electron density $\left(N_{\mathrm{e}}\right)$ inferred from the spin-averaged $(\sim 3 \mathrm{~s}$ resolution) spacecraft potential measured by the electric field instrument (Bonnell et al. 2008) and electrostatic analyzer measurement (McFadden et al. 2008) and (2) spin-averaged magnetic field vector samples acquired by fluxgate magnetometer (Auster et al. 2008) on board THEMIS-A, D, and E probes (Angelopoulos 2008) during 7 years from 2008 to 2014 . This 7 -year period covered the minimum of solar cycle 23 and the rising phase and peak of solar cycle 24 . To examine the spatial distribution of equatorial Alfvén velocity, we used the magnetic field and $N_{\mathrm{e}}$ data acquired from THEMIS probes when the spacecraft were near the magnetic equator between $-5^{\circ}$ and $5^{\circ}$ in magnetic latitude (MLAT).

\section{Results}

\section{Example}

Figure 1a shows the orbit of THEMIS-A projected onto the solar magnetic $x-y$ plane for the 24-h interval on 25 December 2012 with the heavy portion indicating the time period from 0700 to 1500 UT. For this time interval, THEMIS-A was near the magnetic equator with a MLAT between $-1.7^{\circ}$ and $4.4^{\circ}$, and it moved outward from $L=3.2$ in the morning (MLT =9.2) to $L=11.3$ in the afternoon (MLT $=13.1)$. The magnetic field intensity and electron density $N_{\mathrm{e}}$ for the interval of $0700-1500$ UT are shown as a function of $L$ in Fig. $1 \mathrm{~b}$ and c, respectively. The magnetic field intensity decreases monotonically with $L$.

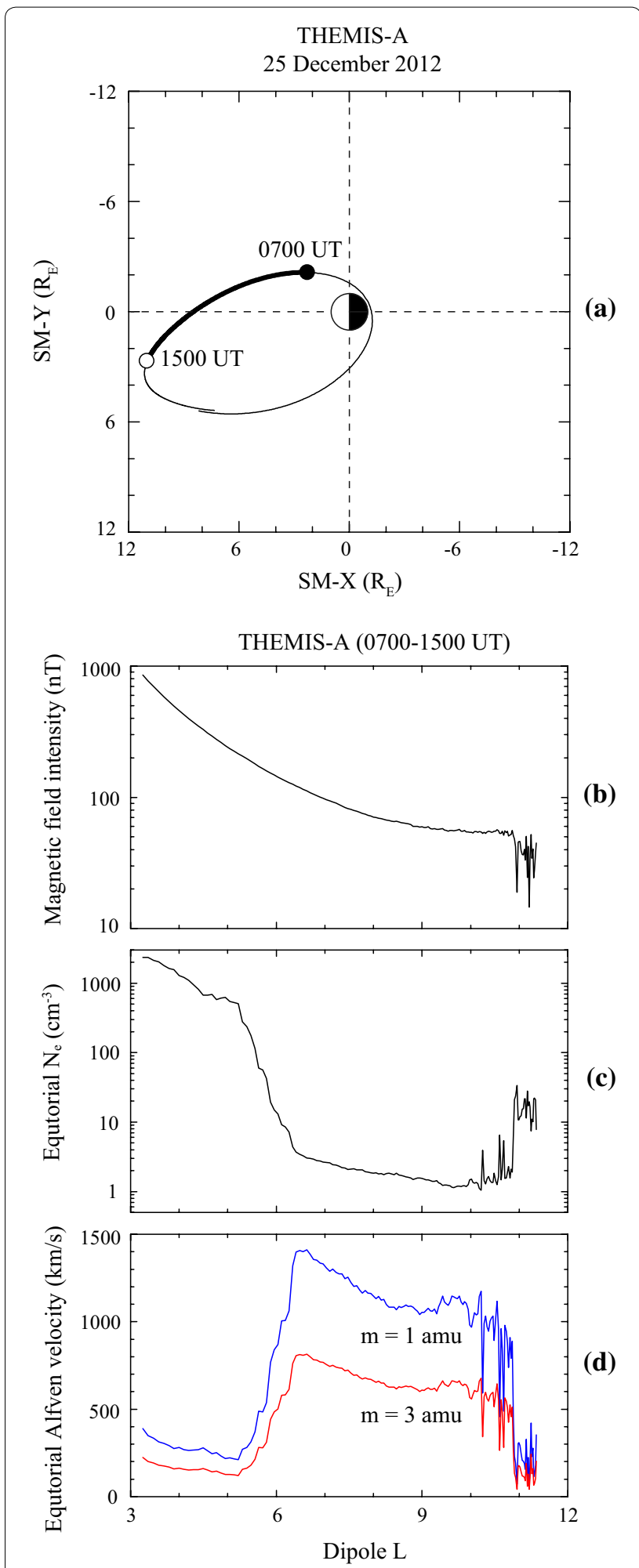

Fig. 1 a The orbit of THEMIS-A projected onto the solar magnetic $x-y$ plane for the 24-h interval on 25 December 2012. b, c The magnetic field intensity and electron density as a function of $L$ for the interval of 0700-1500 UT. d Equatorial Alfvén velocity using Eq. (1) with $m=1$ amu (blue) and $m=3 \mathrm{amu}$ (red) near the magnetic equator 
There is a rapid change in $N_{\mathrm{e}}$ from $\sim 505 \mathrm{~cm}^{-3}$ at $L=5.2$ to $\sim 4 \mathrm{~cm}^{-3}$ at $L=6.4$, indicating that the spacecraft crossed the plasmapause. The plasmapause is located more outward than the typical plasmapause location ( $L \sim 4-5)$. This is due to the quiet geomagnetic conditions on 25 December 2012 (Kwon et al. 2015). Note that the $K p$ index was 1-for 0300-0600 UT, 0 for 0600-0900 UT, $0+$ for $0900-1200 \mathrm{UT}$, and 0 for 1200-1500 UT on that day. In the region of $L>11$, the magnetic field intensity and electron density show large fluctuations, which are attributed to the fact that the spacecraft was in the magnetosheath after the magnetopause crossing at $L \sim 11$.

Figure $1 \mathrm{~d}$ shows Alfvén velocity $\left(V_{\mathrm{A}}\right)$ near the equatorial region given by

$$
V_{\mathrm{A}}=B\left(\mu_{0} \rho\right)^{-1 / 2}
$$

where $B$ is the magnetic field intensity, $\rho$ is the mass density ( $N_{\mathrm{e}} m, m$-the average ion mass), and $\mu_{0}$ is the permeability of free space. To evaluate $V_{\mathrm{A}}$, we have used two cases for the value of $m: m=1 \mathrm{amu}$ (blue), assuming an all- $\mathrm{H}^{+}$plasma, and $m=3$ amu (red), which is a median value inferred from toroidal wave frequencies over $L=4.5-8.0$ (Takahashi et al. 2006). Inside the plasmasphere, $V_{\mathrm{A}}$ decreases with $L$ and has a minimum in the velocity of $\sim 210 \mathrm{~km} / \mathrm{s}$ for $m=1 \mathrm{amu}$ and $\sim 120 \mathrm{~km} / \mathrm{s}$ for $m=3$ amu near the inner edge of the plasmapause. In the outer magnetosphere $(L>7)$, the velocity also shows a decreasing function of $L$ with a maximum of $\sim 1410 \mathrm{~km} / \mathrm{s}$ for $m=1 \mathrm{amu}$ and $\sim 810 \mathrm{~km} / \mathrm{s}$ for $m=3 \mathrm{amu}$ at $L \sim 6.7$. We note that there is solar cycle dependence of the average ion mass: $m=\sim 1 \mathrm{amu}$ during solar minimum and $m=\sim 3-4$ amu during solar maximum (Nosé et al. 2009; Denton et al. 2011).

\section{Statistical analysis}

Figure 2a shows the distribution of total observation time of THEMIS probes (A, D, and E), projected onto the solar magnetic (SM) $x-y$ plane, near the magnetic equator $\left(|\mathrm{MLAT}| \leq 5^{\circ}\right)$ from 2008 to 2014 for all $K p$ values with five concentric gray circles plotted at $4,6,8,10$, and 12 $R_{E}$. The color key indicates the total observation time in each square bin with a size of $1 R_{E} \times 1 R_{E}$. The data coverage depends on the radial distance. That is, there are more observations at larger $L$. This is due to the fact that the spacecraft stayed longer near their apogees $\left(\sim 11 R_{E}\right)$. Figure $2 \mathrm{~b}-\mathrm{d}$ shows the total observation time under different geomagnetic conditions: quiet $(K p \leq 1+)$, moderate $(2-\leq K p \leq 3+)$, and disturbed $(K p \geq 4)$ intervals, respectively. Note that the color key scale in Fig. $2 b-d$ is different from that in Fig. 2a for all $K p$.

Figure $3 a-d$ shows the median amplitudes of the magnetic field intensity $\left(B_{T}\right)$ in logarithmic scale for all $K p$ and under three different geomagnetic conditions. The spatial patters of median $B_{T}$ for all- $K p$ and low/moderate- $K p$ cases are similar to one another, characterized by large magnitude on the dayside and small magnitude on the nightside. Such a day-night asymmetry in $B_{T}$ is due to a dipole like magnetic field configuration on the dayside and a tail-like configuration on the nightside. Under disturbed geomagnetic conditions, the median $B_{T}$ is enhanced at all local times in the outer magnetosphere $(L>6)$. This is due to the superposition of magnetospheric currents, including the magnetopause current, ring current, and tail current, enhanced during disturbed times.

Figure 3e-h shows the median electron density $\left(N_{\mathrm{e}}\right)$ for four levels of $K p$. The most notable feature in the $N_{\mathrm{e}}$ distribution map for all-Kp conditions is the strong dawndusk asymmetry. That is, the overall median density $N_{\mathrm{e}}$ is higher in the duskside outer magnetosphere $(L=6-12)$ than in the dawnside outer magnetosphere. This high density region corresponds to the plasmaspheric bulge (e.g., Carpenter 1970; Gallagher et al. 2000; Goldstein et al. 2004). Such a bulge structure rotates westward
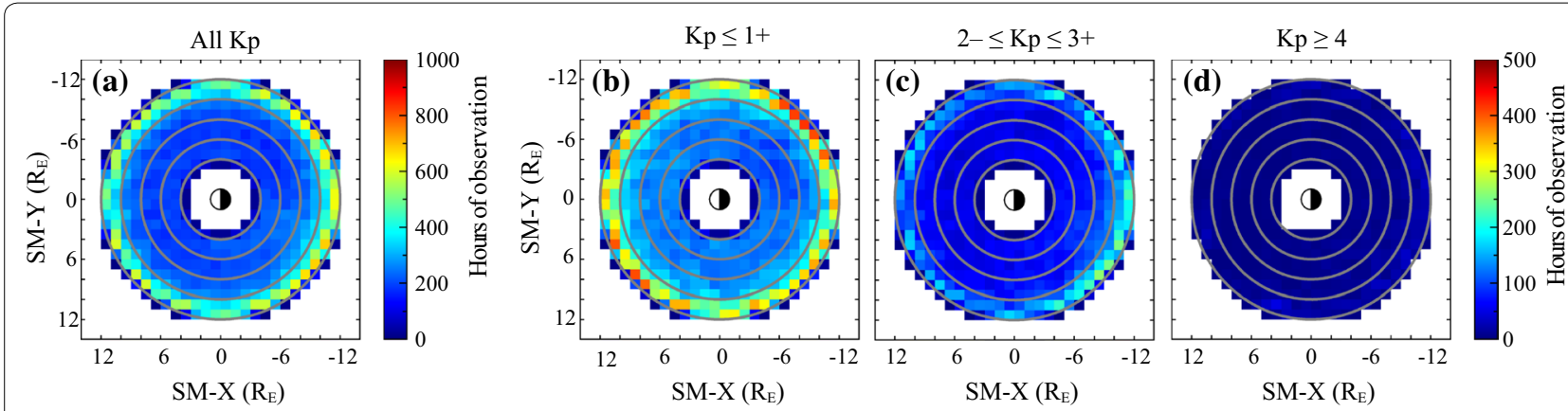

Fig. 2 a The distribution of total observation time of THEMIS probes (A, D, and E), projected onto the solar magnetic (SM) $x-y$ plane, near the magnetic equator $\left(|\mathrm{MLAT}| \leq 5^{\circ}\right)$ from 2008 to 2014. b-d The total observation time under different geomagnetic conditions: quiet $(K p \leq 1+)$, moderate $(2-\leq K p \leq 3+)$, and disturbed $(K p \geq 4)$ intervals, respectively 

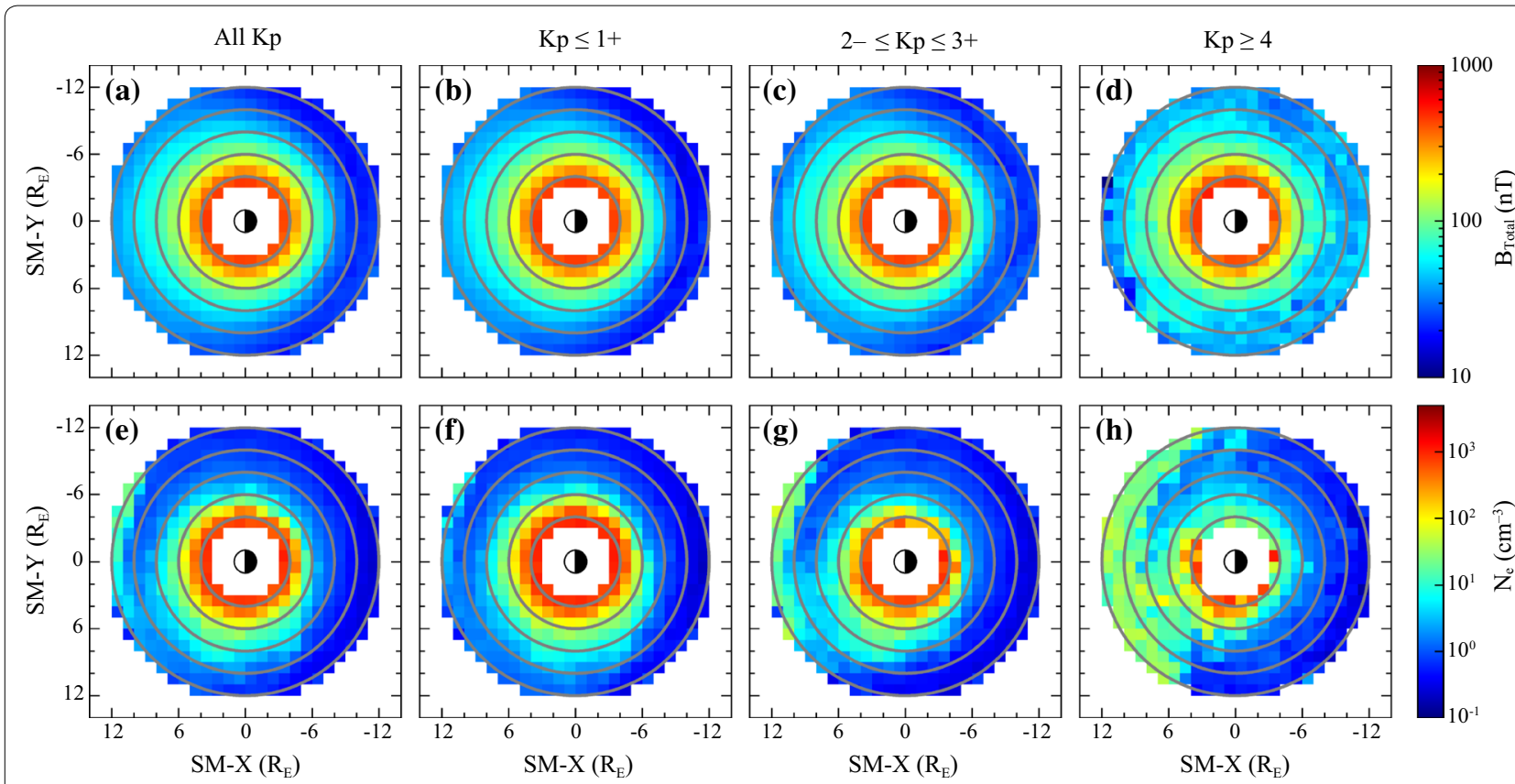

Fig. 3 a-d The median amplitudes of the magnetic field strength $\left(B_{T}\right)$ in logarithmic scale for all $K p$ and under three different geomagnetic conditions. $\mathbf{e}-\mathbf{h}$ The median electron density $\left(N_{\mathrm{e}}\right)$ for all $K p$ and under three different geomagnetic conditions

with the increasing geomagnetic activity. Note that $N_{\mathrm{e}}$ is higher in the outer most region $(L>10)$ near noon under moderate geomagnetic conditions. This high density indicates the magnetosheath density outside the magnetopause. For disturbed geomagnetic conditions $(K p \geq 4)$ the inner edge of the magnetosheath (i.e., the magnetopause) on the dayside moves inward in a region of $L=8 \sim 10$, and the plasmaspheric bulge is attached at the magnetopause in the afternoon sector. The high density at $L=4-6$ shown under quiet conditions disappears as increasing geomagnetic activities. This is due to inward shift of the plasmapause during geomagnetic disturbance intervals.

The equatorial Alfvén velocity $\left(V_{\mathrm{A}}\right)$ is calculated from the magnetic field intensity and electron density observed near the equator using Eq. (1) with $m=1 \mathrm{amu}$ and $m=3 \mathrm{amu}$. The median $V_{\mathrm{A}}$ values of this calculation are shown in Fig. 4 for four $K p$ levels. Note that the linear amplitude scale of the median values for $m=1 \mathrm{amu}$ shown on the right is different from that for $m=3 \mathrm{amu}$. There are three important points to note for the equatorial $V_{\mathrm{A}}$ distribution. First, there is a deep minimum of $V_{\mathrm{A}}(\sim 300-450 \mathrm{~km} / \mathrm{s}$ for $m=1 \mathrm{amu}$ and $200-300 \mathrm{~km} / \mathrm{s}$ for $m=3 \mathrm{amu}$ ) at $L<6$ around all local times for all$K p$ and quiet times. This region corresponds to a steep density gradient region of the plasmapause. Second, a low $V_{\mathrm{A}}$ region extends toward the outer magnetosphere $(L>6)$ in the dusk sector $(\sim 16-20 \mathrm{MLT})$ for all-Kp and quiet times. With increasing $K p$, the low $V_{\mathrm{A}}$ region in the dusk sector rotates toward noon. As expected from the spatial distribution of $N_{\mathrm{e}}$, the pronounced dawn-dusk asymmetry in $V_{\mathrm{A}}$ is mainly due to the local time asymmetry of $N_{\mathrm{e}}$ structure, associated with the plasmaspheric bulge. Third, $V_{\mathrm{A}}$ exhibits a peak in the post-midnight sector $(\sim 00-07 \mathrm{MLT})$ with values of $\sim 1400-1500 \mathrm{~km} / \mathrm{s}$ for $m=1 \mathrm{amu}$ and $\sim 850-900 \mathrm{~km} / \mathrm{s}$ for $m=3 \mathrm{amu}$ at $L=\sim 6$ under moderate conditions. This indicates that the radial gradient of $V_{\mathrm{A}}$ in the outer magnetosphere is larger in the dawn sector than in the dusk sector.

To confirm this asymmetry, we plot the $L$ profiles of $V_{\mathrm{A}}$ medians with the lower and upper quartiles obtained on the dawnside (MLT $=05-07)$ and duskside $(\mathrm{MLT}=17-19)$ with the equatorial plasma mass $m=3$ amu for moderate $(2-\leq K p \leq 3+)$ and quiet $(K p \leq 1+)$ times in Fig. 5. The median $V_{\mathrm{A}}$ in the dawn sector under moderate conditions (Fig. $5 \mathrm{a}$ ) shows a peak value of $\sim 810 \mathrm{~km} / \mathrm{s}$ at $L=6.5$ and decreases with increasing radial distance in the outer magnetosphere $(L>7)$. However, the median $V_{\mathrm{A}}$ in the dusk sector (Fig. 5b) is an increasing function of $L$ with the velocities of $210 \mathrm{~km} / \mathrm{s}$ at the inner limit, $L=4.5$, and of $510 \mathrm{~km} / \mathrm{s}$ at the outer limit, $L=11.5$. Under quiet conditions, the $V_{\mathrm{A}}$ peak in the dawn sector (Fig. 5c) is $550 \mathrm{~km} / \mathrm{s}$, which is smaller than the peak velocity under moderate conditions, at $L=7.5$ and monotonically decreases with the radial distance at $L>8$. The duskside $V_{\text {A }}$ profile (Fig. $5 \mathrm{~d}$ ) 

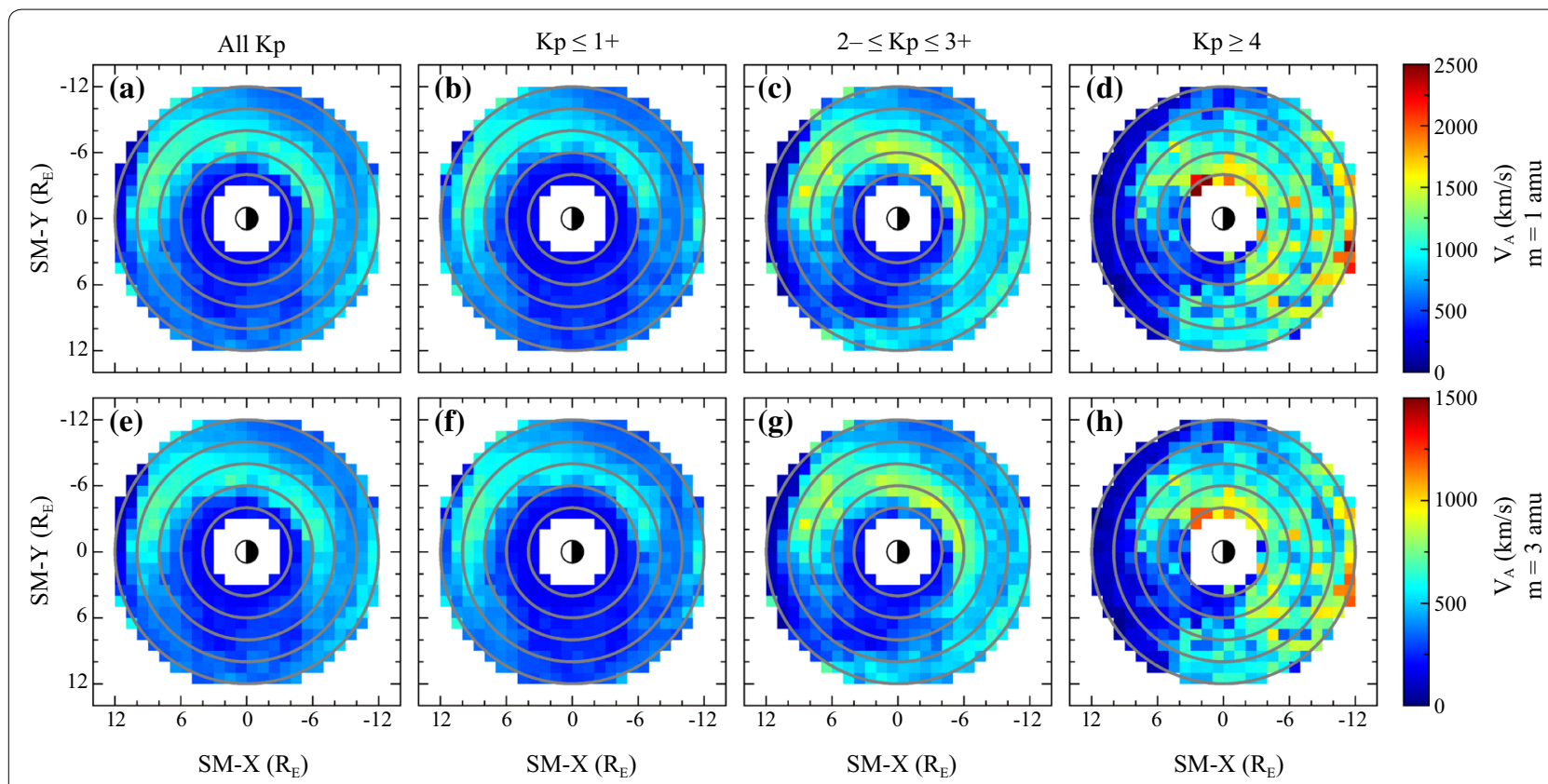

Fig. 4 The equatorial median Alfvén velocity calculated from the magnetic field intensity and electron density observed near the equator using Eq. (1) with $m=1$ amu (a-d) and $m=3$ amu (e-h) for four Kp levels
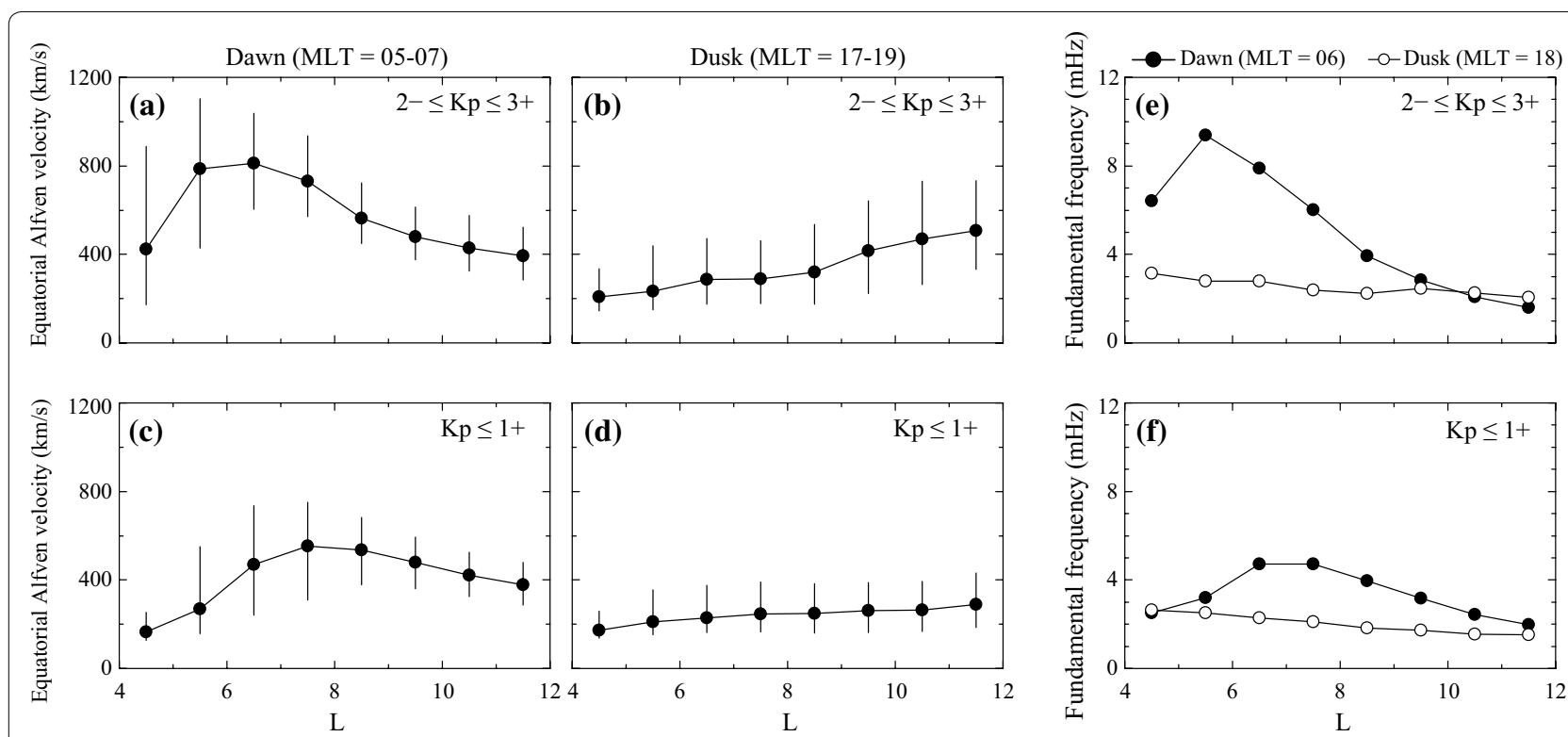

Fig. 5 a-d The $L$ profiles of $V_{A}$ medians obtained on the dawnside (MLT $\left.=05-07\right)$ and duskside $(M L T=17-19)$ with the equatorial mass density $m=3$ amu under moderate and quiet conditions. The dots connected by a straight line indicate the $V_{\mathrm{A}}$ medians, and the vertical bars connect the lower and upper quartiles. e-f The fundamental toroidal Alfvén frequencies at dawn and dusk under moderate and quiet geomagnetic conditions

exhibits a gradual increase with $L$ from $170 \mathrm{~km} / \mathrm{s}$ at $L=4.5-290 \mathrm{~km} / \mathrm{s}$ at $L=11.5$. These radial mean $V_{\mathrm{A}}$ profiles going down in the dawn sector and going up in the dusk sector in the outer magnetosphere are similar to the results of Archer et al. (2015), who used the model magnetic field and electron density obtained from the THEMIS spacecraft without consideration of geomagnetic conditions. In Archer et al. (2015), a peak value of the mean $V_{\mathrm{A}}$ is $\sim 600 \mathrm{~km} / \mathrm{s}$, which is smaller than that in our study, at $L \sim 6$ in the dawn sector. This 
lower $V_{\mathrm{A}}$ is due to the assumption of a higher plasma mass, $m=6.8 \mathrm{amu}$.

The fundamental toroidal Alfvén frequency $\left(f_{\mathrm{T} 1}\right)$ at dawn and dusk under moderate and quiet geomagnetic conditions are plotted in Fig. 5e and f, respectively. The $f_{\mathrm{T} 1}$ is estimated from the median $V_{\mathrm{A}}$ and the field line length obtained from the TS05 model (Tsyganenko and Sitnov 2005). Under moderate (quiet) conditions, $f_{\mathrm{T} 1}$ at dusk decreases gradually from $\sim 3 \mathrm{mHz}$ at $L=4.5(\sim 2.5 \mathrm{mHz}$ at $L=4.5)$ to $\sim 2 \mathrm{mHz}$ at $L=11.5(\sim 1.5 \mathrm{mHz}$ at $L=11.5)$, while $f_{\mathrm{T} 1}$ at dawn decreases rapidly from $\sim 10 \mathrm{mHz}$ at $L=5.5(\sim 5 \mathrm{mHz}$ at $L=7.5)$ to $\sim 1.5 \mathrm{mHz}$ at $L=11.5$ $(\sim 2 \mathrm{mHz}$ at $L=11.5)$. This dawn-dusk difference of $\mathrm{f}_{\mathrm{T} 1}$ is similar to the results presented by Archer et al. (2015) and Takahashi et al. (2016).

\section{Discussion and conclusion}

By using the magnetic field and electron density observed in the $L$ range of $\sim 4-12$ and at all local times near the magnetic equator, we obtain the spatial distribution of equatorial $V_{\mathrm{A}}$. One outstanding feature of the spatial $V_{\mathrm{A}}$ distribution is a pronounced local time asymmetry: The $V_{\mathrm{A}}$ in the prenoon sector is larger than that in the postnoon sector. This is due to the plasmaspheric bulge formed in the afternoon or dusk sector. It is confirmed in Figs. 4 and 5 that the dawn-dusk asymmetry of the plasma density causes qualitatively the similar asymmetry for $V_{\mathrm{A}}$ in the magnetosphere.

We note that our study is not the first to report the spatial distribution of $V_{\mathrm{A}}$. Moore et al. (1987) reported the equatorial $V_{\mathrm{A}}$ distribution, which is calculated using empirical models of the average magnetic field intensity and plasma density. In Moore et al., there is a deep minimum in $V_{\mathrm{A}}$ less than $400 \mathrm{~km} / \mathrm{s}$. Such low $V_{\mathrm{A}}$ values are confined to a region between $L=5$ and $L=6$ in the 12-24 MLT sector. This $V_{\mathrm{A}}$ distribution based on the models is significantly different from our result, using the observed magnetic field and plasma density. As shown in Figs. 4 and 5, the bulge region extends to the duskside outer magnetosphere $(L>6)$, and the low $V_{\mathrm{A}}$ region in the dusk sector extends far out of $L=5-6$. Using the electron density data from THEMIS and a model magnetic field, Archer et al. (2015) examined the radial profile of Alfvén velocity for the dawn (5-7 MLT), noon (11-13 MLT), and dusk (17-19 MLT) sectors and showed a dawn-dusk asymmetry in $V_{\mathrm{A}}$ (see Fig. 1 in their study), which is similar to our results. However, a global map of the equatorial $V_{\mathrm{A}}$ distribution was not provided in Archer et al. (2015).

Matsuoka et al. (1995) reported that compressional Pi3 pulsations excited near the dayside magnetopause by small scale solar wind dynamic pressure variations propagate faster to the morning than to the afternoon in the magnetosphere (see Fig. 7 in their study). They observed $\sim 2$-min time lag between noon and dusk near geosynchronous orbit, corresponding to an average fast mode wave front velocity of $\sim 350 \mathrm{~km} / \mathrm{s}$. Since this value is much smaller than the equatorial Alfvén velocity of $\sim 1100-1700 \mathrm{~km} / \mathrm{s}$ estimated in the prenoon sector (MLT =09-12) by Takahashi and Anderson (1992), Matsuoka et al. (1995) did not consider the observed 2-min time lag as fast mode propagation time near geosynchronous orbit in the dusk sector and suggested that the $\mathrm{Pi} 3$ pulsations propagating duskward are launched at the magnetopause at some point away from the subsolar point. In the present study, we show a strong dawn-dusk asymmetry in $V_{\mathrm{A}}$. As mentioned above, the low $V_{\mathrm{A}}$ region in the dusk-afternoon sector is associated with the plasmaspheric bulge. Since this region is populated with cold plasma, the Alfvén velocity is a good approximation to the fast mode velocity in the plasmaspheric bulge. Thus, the fast mode velocity of $350 \mathrm{~km} / \mathrm{s}$ near geosynchronous orbit in the dusk sector is not an unreasonable value as shown in Fig. 5.

Another notable feature of the $V_{\mathrm{A}}$ spatial distribution is that $V_{\mathrm{A}}$ decreases with $L$ in the dawn sector at $L>7$ and increases with $L$ in the dusk sector at $L=4-11$. That is, the radial $V_{\mathrm{A}}$ gradient, $\mathrm{d} V_{\mathrm{A}} / \mathrm{d} L$, is negative (inward gradient) at dawn and positive (outward gradient) at dusk. In the theory of field line resonance (FLR), the radial $V_{\mathrm{A}}$ gradient is the primary factor contributing to the coupling between the fast mode waves and the FLR oscillations (Kivelson and Southwood 1986; Zhu and Kivelson 1988). The theoretical studies have described that the radial $V_{\mathrm{A}}$ gradient should be negative for energy absorption of fast mode waves by a FLR wave. The gradient parameters related to the FLR efficiency is also estimated by $\mathrm{d} f_{\mathrm{T} 1} / \mathrm{d} L$ (Takahashi et al. 2016). As shown in Fig. 5 , $\mathrm{d} f_{\mathrm{T} 1} / \mathrm{d} L$ is larger at dawn than at dusk, indicating that FLR is stronger at dawn. This FLR property explains why the occurrence rate and amplitude of Pc5 pulsations are higher at dawn than at dusk in the outer magnetosphere (e.g., Anderson et al. 1990; Nosé et al. 1995; Takahashi et al. 2015, 2016).

In conclusion we observed a pronounced dawn-dusk asymmetry of the equatorial $V_{\mathrm{A}}$ in the magnetosphere. That is, $V_{\mathrm{A}}$ at dawn is faster than that at dusk. This asymmetry is mainly due to the duskside bulge in the plasmasphere. According to theoretical and numerical studies, $V_{\mathrm{A}}$ is one of major parameters to determine MHD wave generation and propagation and also degree of FLR. This indicates that model and theory are unable to provide a full explanation for the observations without a realistic $V_{\mathrm{A}}$ distribution. We expect that our observational results make new contribution to numerical and theoretical studies to improve our understanding of the generation and propagation of MHD waves. 


\section{Abbreviation}

$N_{e}$ : electron density; $V_{A}$ : Alfvén velocity; FLR: field line resonance; MLAT: magnetic latitude; MLT: magnetic local time.

\section{Authors' contributions}

KK performed the statistical data analysis and draft the manuscript. GK performed the statistical data analysis. HK contributed to make computer codes for data analysis. All authors read and approved the final manuscript.

\section{Author details}

${ }^{1}$ School of Space Research, Kyung-Hee University, Gyeonggi, Korea. ${ }^{2}$ Division of Polar Climate Sciences, Korea Polar Research Institute, Incheon, Korea.

\section{Acknowledgements}

THEMIS data used in this study are available from Space Science Laboratory, University of California, Berkeley (http://themis.ssl.berkeley.edu). This work was supported by BK21 + through the National Research Foundation (NRF) funded by the Ministry of Education of Korea. The work of K.-H. Kim was supported by the Basic Science Research Program through NRF funded by NRF-2016R1A2B4011553 and also supported by Project PE18020 of the Korea Polar Research Institute.

\section{Competing interests}

The authors declare that they have no competing interests.

\section{Availability of data and materials}

The computer code converting the spacecraft potential to the electron density has been provided from the THEMIS website (http://themis.ssl.berke ley.edu/software.shtml).

\section{Consent for publication}

Not applicable.

Ethics approval and consent to participate

Not applicable.

\section{Funding}

This work was supported by BK21 + through the National Research Foundation (NRF) funded by the Ministry of Education of Korea. The work of K.-H. Kim was supported by the Basic Science Research Program through NRF funded by NRF 2016R1A2B4011553 and also supported by project PE18020 of the Korea Polar Research Institute.

\section{Publisher's Note}

Springer Nature remains neutral with regard to jurisdictional claims in published maps and institutional affiliations.

Received: 21 August 2018 Accepted: 29 October 2018

Published online: 06 November 2018

\section{References}

Anderson BJ, Engebretson MJ, Rounds SP, Zanetti LJ, Potemra TA (1990) A statistical study of PC3-5 pulsations observed by the AMPTE/CCE magnetic field experiment, 1. Occurrence distributions. J Geophys Res 95(A7):10495-10523

Angelopoulos V (2008) The THEMIS mission. Space Sci Rev 141:5-34. https:// doi.org/10.1007/s11214-008-9336-1

Archer MO, Hartinger MD, Walsh BM, Plaschke F, Angelopoulos V (2015) Frequency variability of standing Alfvén waves excited by fast mode resonances in the outer magnetosphere. Geophys Res Lett 42:10150-10159. https://doi.org/10.1002/2015GL066683

Auster HU et al (2008) The THEMIS fluxgate magnetometer. Space Sci Rev 141:235-264. https://doi.org/10.1007/s11214-008-9365-9
Bonnell JW, Mozer FS, Delory GT, Hull AJ, Ergun RE, Cully CM, Angelopoulos V, Harvey PR (2008) The Electric Field Instrument (EFI) for THEMIS. Space Sci Rev 141:303

Carpenter DL (1970) Whistler evidence of the dynamic behavior of the duskside bulge in the plasmasphere. J Geophys Res 75:3837-3847

Chen L, Hasegawa A (1974) A theory of long-period magnetic pulsations: 1. Steady state excitation of field line resonance. J Geophys Res 79:10241032. https://doi.org/10.1029/JA079i007p01024

Degeling AW, Rae IJ, Watt CEJ, Shi QQ, Rankin R, Zong Q-G (2018) Control of ULF wave accessibility to the inner magnetosphere by the convection of plasma density. J Geophys Res Space Phys 123:1086-1099. https://doi. org/10.1002/2017JA024874

Denton RE, Thomsen MF, Takahashi K, Anderson RR, Singer HJ (2011) Solar cycle dependence of bulk ion composition at geosynchronous orbit. J Geophys Res 116:A03212. https://doi.org/10.1029/2010JA016027

Gallagher DL, Craven PD, Comfort RH (2000) Global core plasma model. J Geophys Res 105(A8):18819-18833

Goldstein J, Sandel BR, Thomsen MF, Spasojevic M, Reiff PH (2004) Simultaneous remote sensing and in situ observations of plasmaspheric drainage plumes. J Geophys Res 109:A03202. https://doi.org/10.1029/2003JA0102 81

Kivelson MG, Southwood DJ (1986) Coupling of global magnetospheric MHD eigenmode to field line resonances. J Geophys Res 91(A4):4345-4351. https://doi.org/10.1029/JA091iA04p04345

Kwon H-J, Kim K-H, Jee G, Park J-S, Jin H, Nishimura Y (2015) Plasmapause location under quiet geomagnetic conditions $(K p \leq 1)$ : THEMIS observations. Geophys Res Lett 42:7303-7310. https://doi.org/10.1002/2015GL066090

Matsuoka H, Takahashi K, Yumoto K, Anderson BJ, Sibeck DG (1995) Observation and modeling of compressional Pi 3 magnetic pulsations. J Geophys Res 100(A7):12103-12115

McFadden JP, Carlson CW, Larson D, Ludlam M, Abiad R, Elliott B, Turin P, Marckwordt M, Angelopoulos V (2008) The THEMIS ESA plasma instrument and in-flight calibration. Space Sci Rev 141:277-302

Moore TE, Gallagher DL, Horwitz JL, Comfort RH (1987) MHD wave breaking in the outer magnetosphere. Geophys Res Lett 14:1007-1010

Nosé M, lyemori T, Sugiura M, Slavin JA (1995) A strong dawn/dusk asymmetry in PC5 pulsation occurrence observed by the DE-1 satellite. Geophys Res Lett 22(15):2053-2056

Nosé M, leda A, Christon SP (2009) Geotail observaions of plasma sheet ion composition over 16 years: on variations of average plasma ion mass and $\mathrm{O}^{+}$triggering substorm model. J Geophys Res 114:A07223. https://doi. org/10.1029/2009JA014203

Southwood DJ (1974) Some features of field line resonances in the magnetosphere. Planet Space Sci 22:483-491

Takahashi K, Anderson BJ (1992) Distribution of ULF energy $(f<80 \mathrm{mHz})$ in the inner magnetosphere: A statistical analysis of AMPTE CCE magnetic field data. J Geophys Res 97(A7):10751-10773

Takahashi K, Denton RE, Anderson RR, Hughes WJ (2006) Mass density inferred from toroidal wave frequencies and its comparison to electron density. J Geophys Res 111:A01201. https://doi.org/10.1029/2005JA011286

Takahashi K, Hartinger MD, Angelopoulos V, Glassmeier K-H (2015) A statistical study of fundamental toroidal mode standing Alfvén waves using THEMIS ion bulk velocity data. J Geophys Res Space Phys 120:6474-6495. https://doi.org/10.1002/2015ja021207

Takahashi K, Lee D-H, Merkin VG, Lyon JG, Hartinger MD (2016) On the origin of the dawn-dusk asymmetry of toroidal Pc5 waves. J Geophys Res Space Phys 121:9632-9650. https://doi.org/10.1002/2016JA023009

Tsyganenko NA, Sitnov MI (2005) Modeling the dynamics of the inner magnetosphere during strong geomagnetic storms. J Geophys Res Space Phys 110:A03208. https://doi.org/10.1029/2004JA010798

Wright AN, Elsden T, Takahashi K (2018) Modeling the dawn/dusk asymmetry of field line resonances. J Geophys Res Space Phys 123:6443-6456. https ://doi.org/10.1029/2018JA025638

Zhu X, Kivelson MG (1988) Analytic formulation and quantitative solutions of the coupled ULF wave problem. J Geophys Res Space Phys 93(A8):86028612. https://doi.org/10.1029/JA093iA08p08602 